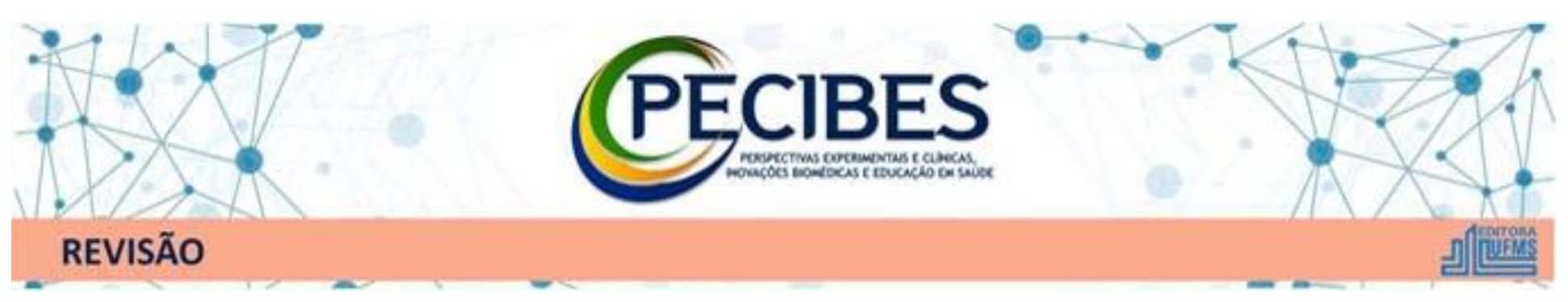

\title{
Inserção percutânea de cateter duplo j: uma breve revisão
}

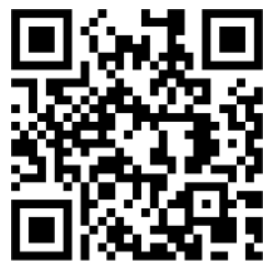

http://www.seer.ufms.br/ind ex.php/pecibes/index

Autor correspondente: Yuree Milhomem Bandeira Herênio, Hospital Universitário Maria Aparecida Pedrossiam Universidade Federal do Mato Grasso do Sul. HUMAP - UFMS E-mail do autor: yureemilhomem@gma il.com

Palavras-chave:

Inserção Percutânea. Cateter Duplo J. Radiologia

Intervencionista.

Obstrução Ureteral.

Key-words:

Percutaneous

Insertion. Double-J

Catheter.

Interventional

Radiology. Ureteral

Obstruction.
Percutaneous insertion of double $\mathrm{j}$ catheter: a brief review

Yuree Milhomem Bandeira Herênio', Rômulo Florêncio Tristão Santos², Tiago Kojun Tibana ${ }^{3}$, Reinaldo Santos Moraes Neto ${ }^{4}$, Thiago Franchi Nunes ${ }^{5}$

${ }^{1}$ Residente de Radiologia e Diagnóstico por Imagem do Hospital Universitário Maria Aparecida Pedrossian - Universidade Federal do Mato Grosso do Sul.

${ }^{2}$ Preceptor e Vice-Coordenador do Serviço de Residência de Radiologia do Hospital Universitário Maria Aparecida Pedrossian - Universidade Federal do Mato Grosso do Sul.

${ }^{3}$ Fellowship de Radiologia Intervencionista do Hospital Universitário Maria Aparecida Pedrossian - Universidade Federal do Mato Grosso do Sul.

${ }^{4}$ Fellowship de Radiologia Intervencionista do Hospital Universitário Maria Aparecida Pedrossian - Universidade Federal do Mato Grosso do Sul.

5 Preceptor e Coordenador do Serviço de Residência de Radiologia do Hospital Universitário Maria Aparecida Pedrossian - Universidade Federal do Mato Grosso do Sul.

Resumo

As intervenções realizadas por radiologistas intervencionistas, guiadas por imagem, mudaram o manejo de várias condições abdominais, particularmente as urológicas. Em razão da sua relação anatômica com os órgãos adjacentes e sua estrutura longa e estreita, os ureteres são facilmente afetados por doenças benignas ou malignas. A inserção percutânea de cateter duplo J é uma técnica alternativa viável e minimamente invasiva. Há poucos estudos na literatura sobre a descrição desta técnica. O objetivo deste artigo de revisão, é pontuar e descrever os principais aspectos relacionados a inserção de cateter duplo J por via percutânea, destacando relatos sobre uma técnica inovadora, suas taxas de sucesso e as complicações mais frequentes.

\section{Abstract}

Image-guided interventions by interventional radiologists have changed the management of various abdominal conditions, particularly urological ones. Due to their anatomical relationship with adjacent organs and their long, narrow structure, ureters are easily affected by benign or malignant diseases. The percutaneous insertion of a double-J catheter is a viable and minimally invasive alternative technique. There are few studies in the literature describing this technique. The purpose of this review article is to point out and describe the main aspects related to percutaneous double-J catheter insertion, highlighting reports on an innovative technique, its success rates and the most frequent complications. 


\section{Introdução}

As intervenções realizadas por radiologistas 2. Material e Métodos

intervencionistas, guiadas por imagem, mudaram o manejo de várias condições abdominais, particularmente as urológicas. Atualmente, continuam a desempenhar papel importante nos procedimentos de drenagem, no manejo da urolitíase, dilatação do sistema pielocalicinal, ablação tumoral e das doenças renovasculares. Múltiplas modalidades de imagem são empregadas para esses propósitos, sendo a fluoroscopia, a ultrassonografia, a tomografia computadorizada, a ressonância magnética e a angiografia com subtração digital as principais. (Tibana et al, 2019).

Em razão da sua relação anatômica com os órgãos adjacentes e sua estrutura longa e estreita, os ureteres são facilmente afetados por doenças benignas ou malignas, resultando na interrupção da drenagem urinária. Na maioria das vezes, a causa da obstrução ureteral é maligna, principalmente por tumores pélvicos, e nesses casos a drenagem da via urinária apresenta maior risco de falha com a abordagem retrógrada (Grubert et al, 2021; Chitale et al, 2002).

A drenagem das vias urinárias pode ser realizada por meio de uma série de técnicas e dispositivos, entre elas a inserção retrógrada por cistoscopia ou percutânea anterógrada do cateter duplo J e nefrostomia percutânea (Nunes et al, 2019).

A inserção de um duplo $\mathrm{J}$ restaura a drenagem urinária fisiológica sem necessidade de um cateter externo (Nunes et al, 2019). Os cateteres utilizados para o tratamento de afecções ureterais obstrutivas são geralmente inseridos por via retrógrada com orientação cistoscópica (Van Der Meer et al, 2017), porém, o procedimento pode não ter sucesso ou ser contraindicado em até $50 \%$ dos pacientes, principalmente quando ocorre obstrução distal ou compressão extrínseca do ureter causada por tumores ( Uthappa et al, 2005). Nessa circunstância, o paciente é tipicamente referido para drenagem percutânea (Nunes et al, 2019).

Não há diretrizes claras sobre os métodos ideais para descompressão do trato urinário no manejo da obstrução ureteral. Os cateteres duplo J (DJ) são em sua maioria inseridos por via retrógrada com orientação cistoscópica (Santos, 2020). Esta abordagem, no entanto, pode ser difícil, especialmente em pacientes com distorção anatômica da parede vesical e obstruções ureterais malignas com comprometimento de longo segmento ureteral, devido à incapacidade técnica em avançar o fio-guia além do ponto de obstrução; em tais casos, as únicas opções são a nefrostomia percutânea ou a inserção de cateter ureteral por abordagem anterógrada (Venyo, 2014).

A inserção anterógrada de cateter duplo J é uma técnica alternativa viável e minimamente invasiva. Há poucos estudos na literatura sobre a descrição desta técnica (Santos, 2020).

O objetivo deste artigo de revisão, é pontuar e descrever os principais aspectos relacionados a inserção de cateter duplo J por via percutânea pela radiologia intervencionista, visto que é uma técnica inovadora e de grande impacto no manejo dos pacientes com obstruções das vias urinárias.

Pacientes que requerem drenagem urinária temporária ou de longo prazo para obstrução do trato urinário superior podem ser manejados por uma série de procedimentos, alguns dos quais incluem inserção de nefrostomia percutânea, inserção anterógrada de cateter, inserção de cateter ureteral retrógrado, construção de conduto ileal, procedimento de ureterostomia, bem como ureteroureteral anastomose (Venyo et al, 2014).

As causas de obstrução urinária podem ser de origem neoplásica primária intrínseca, secundárias extrínsecas de outras malignidades mais comumente de origem ginecológica ou colorretal, com também causas não malignas, ao exemplo da urolitíase e de complicações cirúrgicas (Santos et al, 2020; Nunes et al, 2019).

A nefrostomia e a inserção anterógrada de cateter podem ser realizados com o uso de anestesia local, sedação e analgesia no departamento de radiologia, mas os procedimentos restantes requerem o uso de anestesia geral no centro cirúrgico e exigem que os pacientes estejam em boa forma para serem submetidos à anestesia geral (Venyo et al, 2011).

A nefrostomia percutânea é comumente utilizada como alívio da hidronefrose, especialmente quando há falha na técnica retrógrada. As desvantagens de cateteres de nefrostomia com drenagem externa são o risco de infecção e deslocamento do dreno (Grubert et al, 2021; Bahu et al, 2013). Além disso, os pacientes podem apresentar desconfortos significativos após a inserção de cateter de nefrostomia. Em contrapartida, cateteres duplo J devem ser usados especialmente para situações que requerem um longo período de tratamento para obstruções ureterais (Bahu et al, 2013).

Pacientes submetidos a nefrostomia podem sentir grande desconforto devido a o cateter. Nesse sentido os cateteres DJ podem ser usados alternativamente, especialmente para o tratamento de longo prazo da obstrução ureteral. Os cateteres DJ são geralmente inseridos por via retrógrada com orientação cistoscópica (Chitale et al, 2002). A colocação retrógrada, entretanto, pode ser difícil ou mesmo impossível, especialmente em pacientes com malignidades obstrutivas. Nessas situações a inserção percutânea do cateter duplo J (Figura 1 e 2) pode ser utilizada como técnica alternativa (Van Der Meer et al, 2017). 


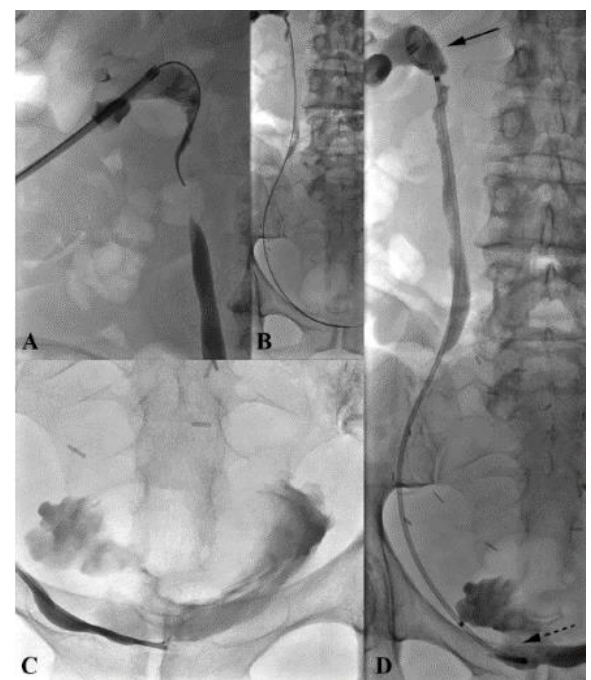

Figura 1 - Inserção percutânea de cateter duplo J. A: Pielografia realizada com inserção de um introdutor 9F. B: Cateterismo do ureter até a bexiga urinária com guia hidrofílico e com cateter 4 ou 5F. C: Confirmação da posição do cateter utilizando meio de contraste radiopaco. D: Posição final do cateter duplo J. (Adapatado de Van Der Meer et al, 2017).

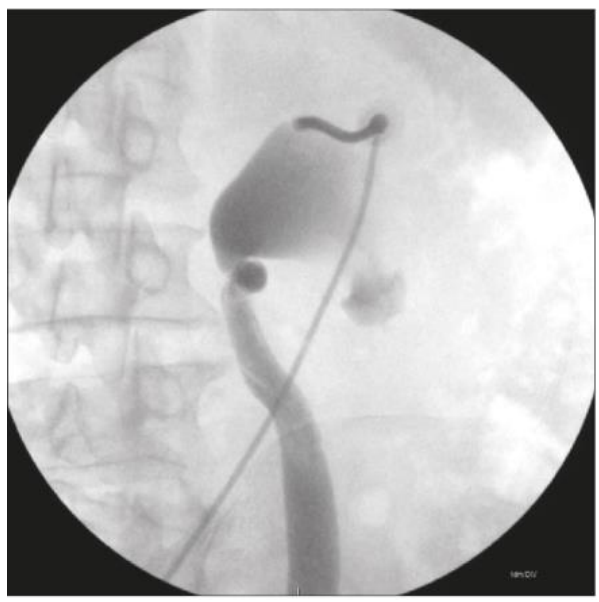

Figura 2 -Punção realizada pelo cálice posterior do sistema coletor superior do rim direito (Santos et al, 2020).

A inserção percutânea do cateter duplo J, utilizando a ultrassonografia e fluoroscopia, pode ser realizada como uma boa alternativa a nefrostomia percutânea e inserção retrógrada do cateter em pacientes com dilatação do sistema coletor renal. Apresenta alta taxa de sucesso, entretanto, é uma técnica pouco difundida. As vantagens desse procedimento incluem menor potencial de complicações, além de ser realizado sob anestesia local e sedação, minimizando os riscos de adversidades pósanestesia geral, principalmente em pacientes graves. Deve idealmente ser realizada por radiologistas intervencionistas com treinamento em procedimentos percutâneos (Nunes et al, 2019; Uthappa et al, 2005).

$\mathrm{O}$ acesso percutâneo ao sistema coletor geralmente é realizado com o paciente em decúbito dorsal, com orientação da fluoroscopia, ultrassonografia e utilizando-se agulha ecogênica (Figura 3), permitindo, assim, a visualização da inserção desde a pele até o cálice renal, preferencialmente pelo cálice médio, que oferece um acesso mais fácil à junção ureteropélvica, ou por um cálice do polo inferior, orientado posterolateralmente, que fornece uma rota de punção segura e relativamente avascular, visando a minimizar complicações como sangramentos e pneumotórax. Nos casos de dilatação discreta do sistema coletor, a técnica coaxial com kit de micropunção é preferida. Procede-se a pielografia anterógrada com injeção de contraste iodado e visualização fluoroscópica da anatomia do sistema coletor (Grubert et al, 2021; Santos et al, 2020; Nunes et al, 2019).

Uma vez que o acesso tenha sido estabelecido, um fio-guia hidrofílico e um cateter diagnóstico 6F são introduzidos sob fluoroscopia pelo sistema coletor até a bexiga. Dessa forma, retira-se a cateter diagnóstico $6 \mathrm{~F}$ da bexiga e prossegue-se com a passagem do Duplo J pelo fio-guia hidrofílico ou rígido (Grubert et al, 2021).

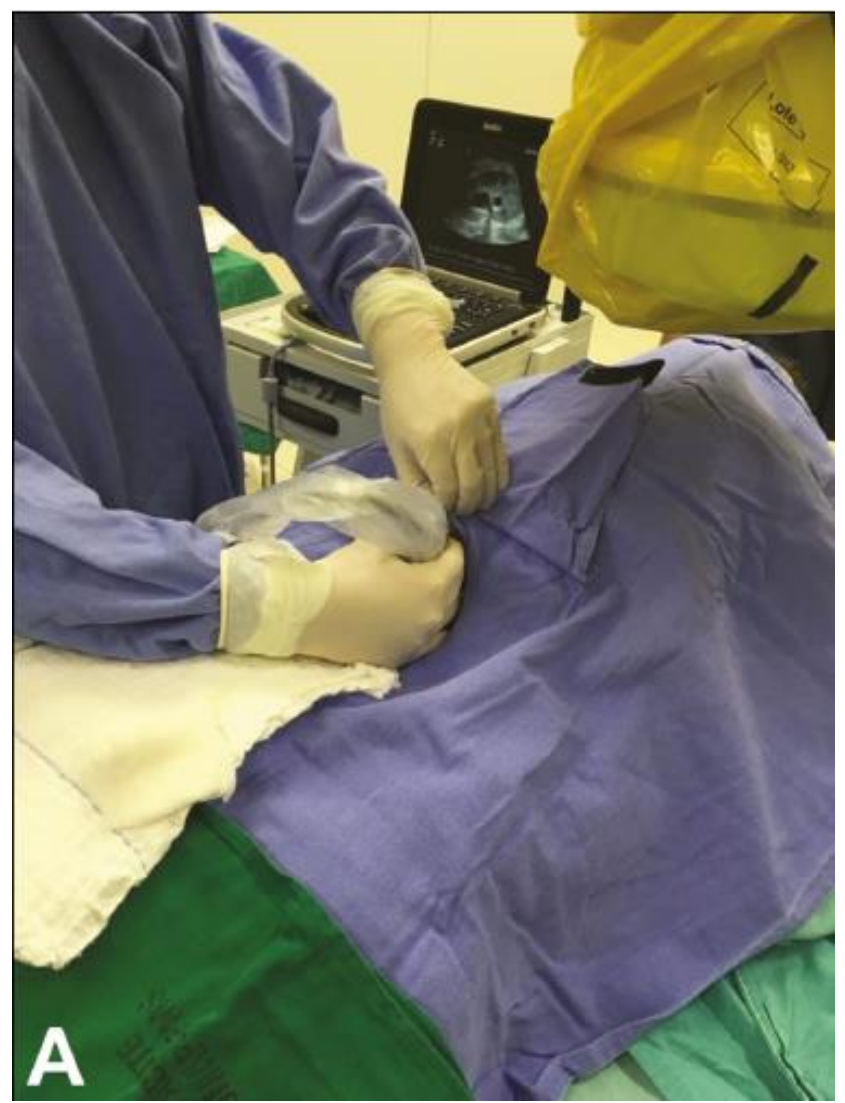

Figura 3 - A: Planejamento do procedimento de inserção percutânea de cateter duplo J com realização de ultrassonografia das vias urinárias na sala de hemodinâmica (Nunes et al, 2019).

O sucesso técnico do procedimento é definido como a manutenção da perviedade da via urinária com redução do grau de hidronefrose, por meio de exames de imagem (ultrassonografia ou tomografia computadorizada) (Figura 4), e o sucesso clínico foi baseado na redução do quadro álgico e dos níveis de escórias nitrogenadas, por acompanhamento via internação e ambulatorial (Santos, 2020; Nunes et al, 2020, Kahriman et al, 2019). 


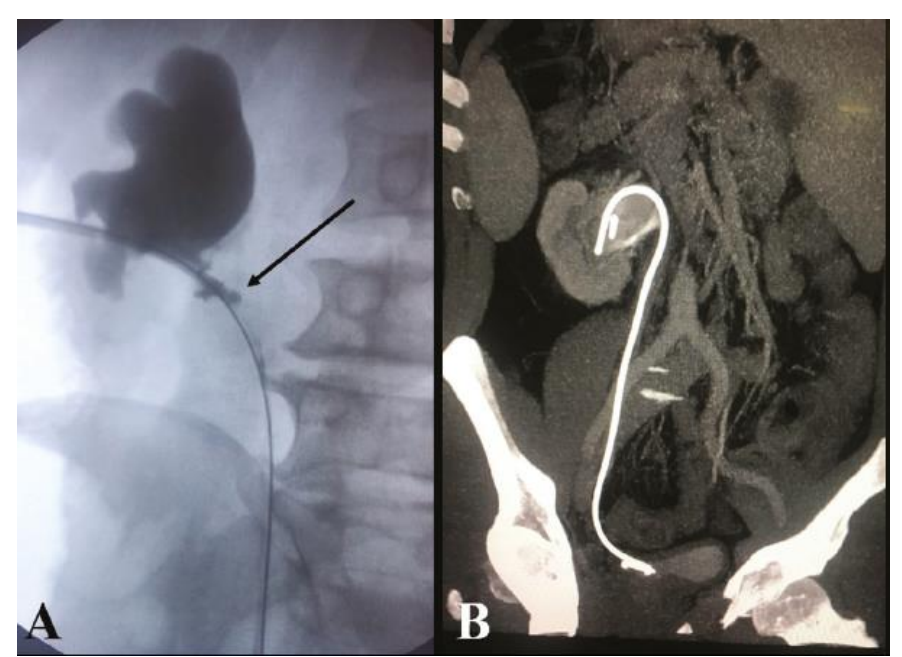

Figura 4. A: Pielografia pré-procedimento de inserção percutânea de cateter duplo J demonstrando estenose do ureter proximal com irregularidades parietais (seta). B: Tomografia computadorizada de abdome coronal pós-contraste, mostrando cateter duplo $\mathrm{J}$ posicionado adequadamente (Nunes et al, 2020).

No estudo retrospectivo realizado em um hospital universitário por Grubert e colaboradores (2021), em 150 pacientes que foram submetidos a inserção anterógrada de cateter duplo $\mathrm{J}$, os procedimentos foram bem sucedidos em 143 pacientes, observando-se 93,5\% de sucesso técnico. Venyo e colaboradores (2014) obtiveram 105 procedimentos com sucesso de um total de 121, taxa de $86,7 \%$. O maior estudo retrospectivo realizado disponível na literatura, foi o de Kahriman e colaboradores (2019), com um total de 461 pacientes incluídos no estudo, obtendo um sucesso técnico de $97 \%$ procedimentos, tabela 1 compara as taxas de sucesso.

\begin{tabular}{|l|l|}
\hline Estudos & Porcentagem \\
\hline Venyo et al. & $86,7 \%$ \\
\hline Grubert el al. & $93,5 \%$ \\
\hline Kahriman et al. & $97 \%$ \\
\hline
\end{tabular}

Tabela 1 - Comparação de sucesso técnico da inserção anterógrada de cateter duplo $\mathrm{J}$ em diferentes estudos.

As complicações do procedimento percutâneo de inserção de Duplo J, são divididas em sistêmicas e locais. A complicação sistêmica mais comum, podendo contribuir para desfechos desfavoráveis é a sepse, isto por que a maioria das obstruções urinárias são potencialmente infectadas, durante a manipulação do sistema urinário com guias e cateteres, a urina infectada pode ser forçada a retornar para a circulação sistêmica. (Hausegger e Portugaller, 2005). A complicações locais mais comuns, são dor local, sangramento, fístulas urinárias e pneumotórax (Hausegger e Portugaller, 2005; Wah et al, 2004).

No estudo realizado por Grubert e colaboradores (2021), analisando retrospectivamente resultados de um estudo com 150 pacientes submetidos a inserção anterógrada de cateter duplo $\mathrm{J}$, as complicações observadas ocorreram em um taxa de $8 \%$ (12 casos), sendo duas por sangramento, e 10 por dor lombar. Venyo e colaboradores (2014), de 121 procedimentos de desobstrução urinária com inserção anterógrada de duplo J, obtiveram taxa de $3,4 \%$ (3 pacientes) de complicação, caracterizada por dor no local de inserção do cateter. De 461 pacientes incluídos no trabalho de Kahriman e colaboradores (2019), as complicações foram: hematoma perirrenal (5), hematúria (3) e abscesso perirrenal (1).

\section{Conclusão}

A inserção anterógrada percutânea de cateter duplo $\mathrm{J}$ em pacientes com obstrução ureteral é eficaz e segura. Na ausência de quaisquer contraindicações clínicas e diante da disponibilidade de equipe de radiologia intervencionista, o implante percutâneo de cateter duplo J pode ser adotado como uma abordagem de rotina para o tratamento da obstrução ureteral maligna e benigna, principalmente para os casos em que houver falha na abordagem retrógrada.

\section{Declaração} interesse.

Os autores declaram que não há conflito de

\section{Referências}

Bahu R, Chaftari AM, Hachem RY, et al. Nephrostomy tube related pyelonephritis in patients with cancer: epidemiology, infection rate and risk factors. J Urol, 189, 130-5, 2013.

Grubert RM, Carmo CEF, Morais Neto RS, Tibana TK, Tristão Santos RF, Marchiori E, Nunes TF. Implante anterógrado de cateter duplo $\mathrm{J}$ como alternativa para abordagem retrógrada: experiência dos primeiros 150 casos em centro único no Brasil. Radiol Bras, in press.

Hausegger KA, Portugaller HR. Percutaneous nephrostomy and antegrade ureteral stenting: techniqueindications-complications. Eur Radiol, 16, 20162030, 2006.

Kahriman G, Özcan N, Doğan A, İmamoğlu H, Demirtaş A. Percutaneous antegrade ureteral stent placement: single center experience. Diagn Interv Radiol, 25, 127-133, 2019.

Nunes TF, Tibana TK, Santos RFT, et al. Percutaneous insertion of bilateral double stent. Radiol Bras, 52, 104-5, 2019.

Nunes TF, Tibana TK, Santos RFT, Faria BB, Fornazari VAV, Marchiori E. Acesso percutâneo no diagnóstico de neoplasias uroteliais: ensaio iconográfico com correlação anatomopatológica. Radiol Bras, 53, 345-348, 2020.

Palanca AB, Puchol F, Fort RV, Romero JG. Inserción anterógrada de catéter ureteral. Actas Urol Esp, 24, 243-247, 2000.

Santos RFT, Tibana TK, Marchiori E, Nunes TF. Inserção anterógrada de cateter duplo $\mathrm{J}$ no tratamento 
deobstruções ureterais malignas: análise retrospectiva dos resultados de uma técnica modificada em um hospital universitário. Radiol Bras, 53, 155-160, 2020.

Skriver EB, Miskowiak J, Mygind T. Percutaneous introduction of double-j ureteral stents. Scand J Urol Nephrol, 21, 47-50, 1987.

Tibana TK, Fornazari VAV, Gutierrez Junior W, Marchiori E, Szejnfeld D, Nunes TF. O que o radiologista deve saber sobre o papel da radiologia intervencionista em urologia. Radiol Bras, 52, 331-336, 2019.

Tibana TK, Grubert RM, Santos RFT, Fornazari VAV, Domingos AA, Reis WT, Marchiori E, Nunes TF. Percutaneous nephrostomy versus antegrade double-J stent placement in the treatment of malignant obstructive uropathy: a costeffectiveness analysis from the perspective of the Brazilian public health care system. Radiol Bras, 52, 305-311, 2019.

Uthappa MC, Cowan NC. Retrograde or antegrade doublepigtail stent placement for malignant ureteric obstruction?. Clinical Radiology, 60, 608-612, 2005.

Venyo A, Bakir E. Antegrade Ureteric Stenting: Prospective Experience In Managing 30 Patients; Indications, Complications And Outcome. Webmed Central Urology, 2, 1-18, 2011.

Venyo AK, Hanley T, Barret M, Khan AN. Ante-grade ureteric stenting, retrospective experience in managing 89 patients: Indications, complications and outcome. Journal of Biomedical Graphics and Computing, 4, 47-56, 2014.

Van Der Meer RW, Weltings S, Van Erkel AR, Roshani H, Elzevier HW, Van Dijk LC, Van Overhagen H. Antegrade Ureteral Stenting is a Good Alternative for the Retrograde Approach. Curr Urol, 10, 87-91, 2016. 